\title{
Research on the Application of Intelligent Algorithm in Short-Term Traffic Flow Forecast
}

\author{
Wang Danping ${ }^{1,2}$, Hu Kunyuan ${ }^{1}$, Han Xiaowei ${ }^{2}$ \\ ${ }^{1}$ Shenyang Institute of Automation Chinese Academy of Sciences, 110016 Shenyang, China \\ ${ }^{2}$ Shenyang University, 110044 Shenyang, China \\ aiamdanping@163.com
}

Keywords: Short-term traffic flow prediction, genetic algorithm.

\begin{abstract}
In recent years, with the vigorous development of intelligent transportation systems, traffic control and traffic flow guidance have become popular issue of intelligent transportation systems (ITS). The key issue to achieve traffic control guidance is to realize real-time and accurate short-term traffic flow forecasting. And the accuracy and real-time of prediction directly impact traffic control and induced effect. Achieving an accurate prediction of urban road short-term traffic flow is the key of urban road traffic control and traffic guidance. Since the single prediction method for the current is of low precision, we proposed wavelet and support vector machine (SVM) method to predict new fusion; in order to avoid falling into local optimal problem in the process while learning SVM knowledge, we use the particle swarm optimization (PSO) to optimize the key parameters of SVM and in order to improve the prediction accuracy of short-term traffic flow.
\end{abstract}

\section{Introduction}

In several important part of intelligent transportation systems, traffic flow state dynamic analysis and forecasting short-term traffic congestion is a very important basic theory is advanced traveler information systems (ATS) and advanced traffic management system (ATMS) core part of the transport sector is one of the world's current problems. So far, experts and scholars around the world in different disciplines using the method developed various methods for predicting short-term traffic flow forecasting. To sum up, probably in the following categories: forecasting based on linear system theory, prediction method based on dynamic traffic assignment, intelligent forecasting model based on knowledge discovery, based on the prediction method of nonlinear system theory and the combination of model-based prediction methods. Given the nonlinear road transport system, the complexity and uncertainty of the basic characteristics of intelligent algorithms based on nonlinear system forecasting model will be the development trend in the field of traffic flow forecasting [1].

Reliable prediction of traffic flow is the key technology of urban road traffic control and traffic guidance optimization. Where short-term traffic flow forecasting is the core of intelligent transport systems, because of the characteristics of short-term traffic flow data with a high degree of nonlinearity and uncertainty, difficult to implement accurate and reliable flow forecast. Short-time traffic flow data with strong chronological is the typical of the time series forecasting problems. Due to the high complexity of road traffic flow, randomness and uncertainty, the traditional forecasting methods have been unable to obtain a satisfactory prediction result, which affected traffic control results in a certain degree. In order to improve traffic flow forecasting accuracy, the method of nonlinear system theory and artificial intelligence methods to get the attention of scholars, including wavelet analysis, empirical mode decomposition, neural networks and support vector machines, these intelligent methods to effectively improve the prediction accuracy.

Given the wavelet analysis can be a good interference suppression nonlinear signal timing, and support vector machine (SVM) model better generalization ability, the advantages of both the organic integration of this article, is proposed based on PSO - Wavelet -SVM city short road traffic flow forecasting methods. Through actual urban road traffic data, experimental study, the results 
showed that the use of relatively simple, the prediction accuracy of the proposed method is better, and SVM based on PSO is better than the performance of neural networks [2].

\section{The importance of short-term traffic flow prediction}

In recent years, with the rapid development of intelligent transportation systems, traffic control and real-time traffic flow guidance become intelligent transportation systems (ITS) studies of topical issues and the realization induced traffic control key issue is real-time and accurate shortterm traffic flow forecast, effective traffic-induced traffic forecast is accurate as a precondition, so traffic flow forecasting has become the focus of attention of domestic and foreign experts. The latest research shows that transportation, traffic prediction in various traffic control system also plays a very important role. Traffic prediction accuracy will directly affect the traffic control and vehicle-induced effects, both for traffic guidance system or traffic control systems, real-time and accurate traffic prediction is the premise and key implementations of these systems, traffic prediction results is directly related to the effect of traffic guidance and control. We can say that accurate real-time traffic flow forecasting is to make intelligent transportation system from a "passive response" changes to "proactive action" key [3].

But we all know, the road traffic system is a people participation, time-varying, complex nonlinear large-scale systems, its distinguishing feature is the high degree of uncertainty, both from the aspect of nature reasons (such as seasonal and climatic factors, etc.), there are reasons from human factors (such as traffic accidents, emergencies, driver's psychological state, etc.), the latter of which is more difficult to estimate. These factors gave the traffic flow makes it difficult to predict, especially short-term traffic flow forecasting greater impact by random factors, uncertainty stronger, more obvious regularity, which is short-term traffic flow prediction with respect to the long-term traffic forecasts difficulty lies.

\section{The review of existing methods of traffic flow forecasting}

Traffic flow forecast is based on the existing traffic flow data, real-time prediction at time t next time $t+\Delta_{t}$, and after some time the traffic flow. Generally believed that short-term traffic flow prediction refers to the forecast period $\Delta_{t}$ is no more than 15 minutes (or even less than 5 minutes) of the traffic flow. As predicted shorter scale, traffic flow showed a high degree of complexity, randomness and uncertainty, which is the short-term traffic flow forecasting more difficult to predict than the long-term causes [4].

Need for real-time dynamic control of the people in the sixties and seventies began to apply in other areas ripe for short-term traffic flow forecasting model to predict the field, reported in the literature has been proposed nearly 30 kinds of prediction methods. Existing short-term traffic flow prediction method can be roughly divided into two categories: the traditional method of mathematics and physics is the first class of mathematical statistics and calculus-based prediction methods, including early historical average models, autoregressive moving average model (ARMA), and then more complex, higher accuracy of the multiple regression model, ARIMA model, Kalman filter model, etc; second category is no predictive mathematical models, including non-parametric regression, spectral analysis, based on wavelet theory method, neural network and so on.

\section{Historical average model}

Historical average model (History Average Model) is the average of the past to predict the future traffic volume of traffic, which uses the cycle characteristics of traffic flow. It is assumed that the traffic situation is occurring intermittently, that have the same historical trends in the various sections of the day with the same traffic flow characteristics at the same time. Historical average model consists of two parts: a section of the road network within a week of each per day for each interval of typical traffic flow data and prediction algorithms. Typically traffic flow data for each 
period of the study period the test results can be obtained from the data updated through the collection of traffic data for each observation point for each interval of time to form the historical database.

Time series algorithms. Initially, only a simple autoregressive model (AR), moving average model (MA), autoregressive moving average model (ARMA) used in traffic flow forecasting, BoxJenkins algorithm later also applied to the time series, which is the autoregressive integrated moving average (ARIMA) method. ARIMA (autoregressive integrated moving average) is a linear time series forecasting model, its basic idea is: the data objects over time series prediction is formed as a random sequence, with certain mathematical model to describe the sequence of approximate. Once this model can be identified the time-series can predict future values from the past and the present value to. ARIMA forecasting model as the one for non-stationary time series, also applied to short-term traffic flow prediction.

Kalman filter algorithm. Kalman filter is an advanced control method, but also a sophisticated forecasting method based on linear regression analysis. It is based on Kalman filtering theory proposed 60 years based on the optimization of data processing regression algorithms. Kalman filter with a wide range of adaptability, because it uses a more flexible recursive state space model, both to deal with smooth data, but also to deal with non-stationary data; as long as the state variables make different assumptions, it can describe and handle different types of problems; model with linear, unbiased minimum mean square error, easy to implement on a computer, and greatly reduces the amount of computer memory and computing time, suitable for on-line analysis; high prediction accuracy. Kalman filtering method development has been there many, such as linear filtering, nonlinear filtering, adaptive filtering, and a variety of other methods to simplify the filtering. However, the use of the Kalman filter parameter vector to be calculated, the need for large amounts of matrix and vector operations, the algorithm is more complicated [5].

Neural network prediction algorithm. In terms of (Neural Network Model) to predict changes in traffic flow using neural networks, the majority of experts and scholars have made a lot of research, the theory is more mature. Neural network in traffic flow forecasting, to a certain extent, out of trouble to establish accurate mathematical model for the study opens up new ideas. In the 1990s, studies abroad in the use of neural networks to predict the emergence of a boom, but also achieved some results, the neural network model is a promising model in traffic prediction [6].

Nonparametric regression algorithm. Nonparametric regression itself born out of chaos theory, originally used to predict the trajectory of the behavior of chaotic systems. The idea is: Find a similar history and current state of the state in the history of the database, and the history and current state of the matching point applies state prediction. It is a non-parametric model of a portable, the prediction accuracy is relatively high, especially for the traffic state is unstable and the reason is that it does not have the historical data for the regression or smoothing, but the use of all possible traffic flow saved status information. The five elements of nonparametric regression are: preparation of historical data, select the state vector selection, similar to the mechanism of selection mechanism to select neighbor, forecasting function. Smith with 95 years in the short-term traffic flow forecasting, but because of its slow speed data search, database usage history to predict the required resources, too, as well as the theoretical system of this algorithm is no system like useless application.

\section{Short-term traffic flow forecasting model based on multi-fusion}

In the external excitation interference, short-term non-stationary traffic showing the different signal components will cause a change in the trend of different traffic characteristics, getting steady traffic signal changes leading the overall trend, time-varying interference signal to flow up and down in the vicinity of the general trend fluctuations, so it is necessary to make the nonlinear factors predictive value close to the real results. Wavelet transform (wavelet transformation, WT) is a study of non-stationary signals a strong time-frequency domain analysis tools emerged in recent years, its core is a multi-resolution analysis, can be at different scales from coarse to fine approach, it can not only reflect the overall characteristics of the signal, but also to reflect the local 
information signal. Since the wavelet transform analysis precision adjustable, which allows both of the short-time high frequency components in the signal for positioning, but also the low-frequency component of the signal analysis, Fourier analysis overcome in the time domain without any resolution of the defect, Fourier transform can extract more detailed information and a short signal. Therefore, the use of multi-WT decomposition on the traffic flow, suppress transient excitations outside influence, each wavelet components more accurately reflect the variation of traffic flow. Then layers of wavelet data reconstruction, SVM prediction model were established for each wavelet components, all the predicted values to get the final cumulative traffic flow prediction [7].

SVM is a statistical learning algorithm proposed by Vapnik in 1995 to solve the small sample, nonlinear and high dimensional pattern recognition, machine learning problems. In the VC dimension theory and structural risk minimization principle on the basis of, SVM with linear discriminant function and the training error as a constraint optimization problem to achieve the best compromise between the limited sample information model complexity and ability to learn, to obtain much better than some of the traditional generalization ability of learning methods. Among them, the least squares support vector machine (LS-SVM) is based on an improved SVM algorithm, using the constraint equation replaces the standard support vector machine inequality constraints, the SVM in the quadratic programming problem is transformed into linear equations solving, simplifying algorithm. Therefore, this study uses LS-SVM intelligently identify failure modes. Detailed derivation and argumentation theory LS-SVM See [10], just to give LS-SVM regression model:

$$
f(x)=\sum_{i=1}^{l} \alpha K\left(x_{i}, x\right)+b
$$

Where: $\alpha$ is the Lagrange multiplier; $\mathrm{b}$ is the bias constant; kernel using RBF kernel:

$$
K\left(x_{i}, x\right)=\exp \left[-\frac{\left|x-x_{i}\right|^{2}}{2^{\sigma^{2}}}\right]
$$

For the RBF kernel SVM, its parameters generalization capabilities include boundary parameters $\mathrm{C}$ and kernel parameter $\sigma$ regression model has a great influence. So this paper, particle swarm optimization (PSO) to optimize the parameters for SVM.

PSO is similar to the genetic algorithm is iterative optimization. But not by crossover and mutation PSO update offspring, but according to their flying experience and particles flying experience to adjust their companions flight [11]. The best position of each particle during the flight experienced, is to find the optimal solution particles themselves, called individual extremum $(\mathrm{Pb})$, the entire group experienced global extremum $(\mathrm{Gb})$ that is currently found in the best of the entire group solution. In PSO velocity $\mathrm{v}$ and position update algorithm $\mathrm{P}$

$$
\begin{aligned}
& v_{i}(k+1)=\omega_{i} v_{i}(k)+c_{i} r_{i}\left[P_{b}-P_{i}(k)\right]+c_{2} r_{2}\left[G_{b}-P_{i}(k)\right] \\
& P_{i}(k+1)=P_{i}(k)+v_{i}(k+1)
\end{aligned}
$$

Wherein: $\omega_{i}$ is the inertia factor; $r_{1}, r_{2}$ for a random number $[0,1]$ among; $c_{1}, c_{2}$ for the learning factor is usually set to two.

Thus, the extracted by organic fusion can establish short-term traffic flow forecasting model based on the integration of multi-method: First, traffic flow information using wavelet pretreatment, different components of the original non-stationary signals to different resolution wavelet decomposition child band, thus separating better reconstructed wavelet sub-signals with different characteristics, effectively inhibit the strong coupling of different signal components; secondly to establish SVM forecasting model of each wavelet sub-sub-signals to separate the different components of the original signal, respectively forecast, thus the original complex signal into a plurality of relatively simple, independent signals can be processed to improve the robustness of the signal prediction; while using PSO algorithm parameters for nuclear boundary parameters R and C each sub-SVM models were optimization further enhance the learning ability and generalization ability sub models for accurate prediction wavelet sub-signal; final results of the SVM prediction 
model obtained by adding sub relatively reliably predict the final outcome [8]. Workflow this prediction system is shown in Figure 1.

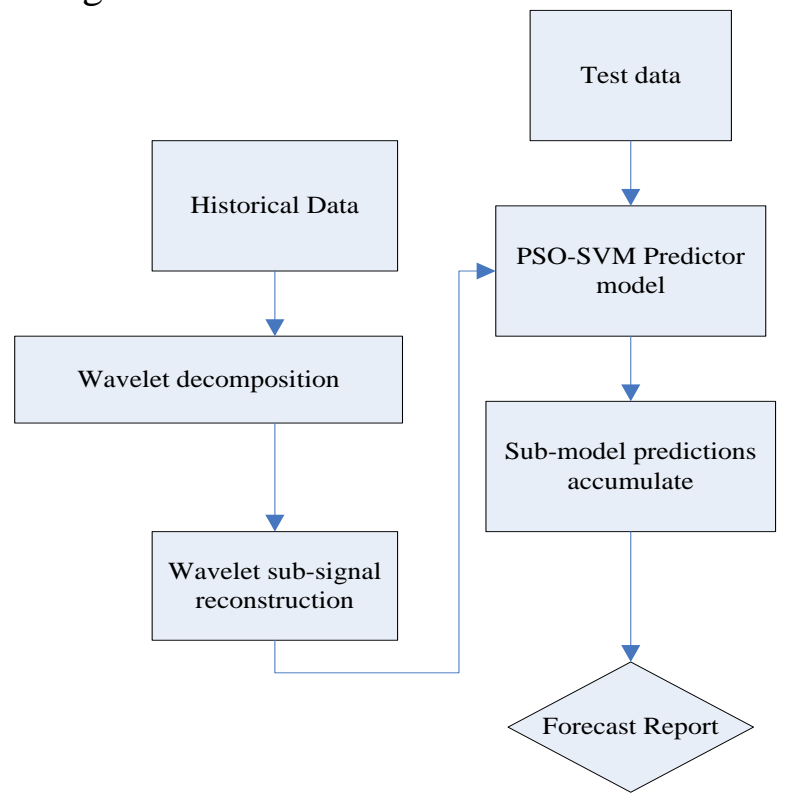

Fig. 1 The workflow of the short time traffic flow predictive model based on multi-method fusion

\section{Experimental data acquisition}

Select a city center area studied. Actual forecasts, traffic statistics using a crossroad of five consecutive days, every 20 min of recording time traffic within that period, a total of 360 times to collect data points, with the former 4d 288 data as SVM training data, with the last $1 \mathrm{~d}$ of 72 data detection performance prediction model.

\section{WT-PSO-SVM forecasting model}

The proposed prediction method firstly gives a wavelet decomposition and reconstruction of the original data. Since this was the establishment of SVM prediction model for wavelet reconstruction for each sub-signal, so in order to reduce the computational complexity of the original signal into two layers wavelet decomposition, wavelet mother function selection / DB10, get the Layer 2 subspace 4 wavelet reconstruction signals. Low-frequency signal reflects the overall trend piece traffic flow collected several other sub-signal represents the uncertainty of information can be a good description of the wavelet decomposition of the raw data to identify characteristics of different ingredients, were established in favor of targeted the SVM sub models. In this paper, PSO-SVM were established four predictor model to predict the result of each sub-model to get through the training and test data, and then get the final urban road traffic flow forecasting short-term value addition. SVM and use of this method were the first $5 \mathrm{~d} 72$ 个 traffic data to predict the curve; Table 1 shows the prediction error SVM, PSO-SVM method and the results of the analysis in this article. WT-PSO-SVM prediction is closer to the true value than just SVM method. The apparent from Table 1, the new prediction methodology wavelet proposed binding with PSO-SVM is the average relative error is higher than the maximum relative error using only SVM prediction, and prediction accuracy than conventional PSO-SVM is better. Therefore, short-term traffic flow prediction method of urban roads in this paper has a high predictive ability to effectively improve the urban road traffic flow forecasting short-term accuracy.

Table.1: The comparison of the prediction performance

\begin{tabular}{lccc}
\hline Diagnostic methods & The average relative error/\% & The maximum relative error/\% & Goodness of fit \\
\hline SVM & 13.9 & 18.1 & 0.89 \\
PSO-SVM & 8.3 & 11.1 & 0.94 \\
WT-PSO-SVM & 4.2 & 6.9 & 0.98 \\
\hline
\end{tabular}




\section{Conclusions}

For quick construction of traffic management information system to achieve traffic intelligent control and induction, we firstly need to predict the short-term traffic flow accurately. However, due to the lots of variability, uncertainty of factors that impact the short-term traffic flow forecasting, it is much more difficult to adopt a particular analytical method for accurate predictions. Therefore, this article combined wavelet decomposition and PSO-SVM to be used in intelligent transportation in urban road short time traffic flow forecasting. Through experimental analysis of actual traffic data, the results show that the proposed prediction method can get higher prediction accuracy than using a separate SVM and with better engineering value.

\section{Acknowledgements}

The research work was supported by Liaoning city of Shenyang province science and technology plan project number: F13-298-1-00.

\section{References}

[1] Dia H. An object-oriented neural network approach to short-term traffic forecasting[J]. European Journal of Operational Research, 131(2), pp.253-261, 2001

[2] Brian L. Smith, Billy M. Williams, Comparison of parametric and nonparametric models for traffic flow forecasting. Transportation Research C, 10(4), pp.303 321, 2002

[3] B. L. Smith, M. J. Demetsky, Traffic flow forecasting: comparison of modeling approaches, ASCE Journal of Transportation Engineering, 123(4), pp.261 266, 2007

[4] Yun S.Y., Namkoong S.,A performance evaluation of neural network models in traffic volume forecasting, Mathematical and Computer Modeling, 27(2), pp.293 310,2008

[5] Hussein D., An objected-oriented neural network approach to short-term traffic forecasting, Special Issue of the European Journal of Operation Research, 131(2), pp.235 261, 2001

[6]Dougherty M S, Short-term inter-urban traffic forecasting using neutralnetwork. International Journal of Forecasting, 24(13), pp.21 31, 2007

[7]G. A. Davis, N.L. Nihan, Nonparametric regression and short-term freeway traffic forecasting, ASCE Journal of Transportation Engineering, 117(2), pp.178 188, 2001

[8]Oswald P K, William T S, Brian L S, Traffic flow forecasting using approximate nearest nonparametric regression. Research Report,No.UVACTA-15-13-17, 2001 